\title{
Numerical Simulation of Deflagration to Detonation Transition in a Straight Duct: Effects of Energy Release and Detonation Stability
}

\author{
Hua-Shu Dou ${ }^{1, *}$, Zongmin $\mathrm{Hu}^{2}$, Boo Cheong $\mathrm{Khoo}^{3}$ \\ and Zonglin Jiang ${ }^{2}$ \\ ${ }^{1}$ Faculty of Mechanical Engineering and Automation, Zhejiang Sci-Tech University, \\ Hangzhou 310018, China \\ 2 State Key Laboratory of High Temperature Gas Dynamics, Institute of Mechanics, \\ Chinese Academy of Sciences, Beijing 100080, China \\ ${ }^{3}$ Department of Mechanical Engineering, National University of Singapore, \\ Singapore 119260, Singapore
}

Received 18 March 2013; Accepted (in revised version) 19 June 2014

Available online 7 August 2014

\begin{abstract}
Numerical simulation based on the Euler equation and one-step reaction model is carried out to investigate the process of deflagration to detonation transition (DDT) occurring in a straight duct. The numerical method used includes a high resolution fifth-order weighted essentially non-oscillatory (WENO) scheme for spatial discretization, coupled with a third order total variation diminishing Runge-Kutta time stepping method. In particular, effect of energy release on the DDT process is studied. The model parameters used are the heat release at $q=50,30,25,20,15,10$ and 5, the specific heat ratio at 1.2 , and the activation temperature at $T i=15$, respectively. For all the cases, the initial energy in the spark is about the same compared to the detonation energy at the Chapman-Jouguet (CJ) state. It is found from the simulation that the DDT occurrence strongly depends on the magnitude of the energy release. The run-up distance of DDT occurrence decreases with the increase of the energy release for $q=50 \sim 20$, and increases with the increase of the energy release for $q=20 \sim 5$. This phenomenon is found to be in agreement with the analysis of mathematical stability theory. It is suggested that the factors to strengthen the DDT would make the detonation more stable, and vice versa. Finally, it is concluded from the simulations that the interaction of the shock wave and the flame front is the main reason for leading to DDT.
\end{abstract}

PACS: 47.40.-x, 47.40.Rs, 47.70.Fw

Key words: Numerical simulation, detonation, DDT, energy release, duct.

${ }^{*}$ Corresponding author.

Email: huashudou@yahoo.com (H.S. Dou) 


\section{Introduction}

Deflagration to detonation transition (DDT) is one of the ways to produce detonation which starts from low speed combustion and then accelerates and transits to detonation under certain conditions. Alternatively, detonation can also be produced by direct ignition which requires sufficiently high energy input. In most cases, detonation is generated by DDT since only low energy is required and also it is easier to control. The understanding of the DDT process is therefore important for implementation and control of the detonation. In recent years, much interest has been focused on this phenomenon because of the direct potential application in the pulse detonation engines [1,2]. On the other hand, it is also useful in disaster prevention and loss prediction [3]. Although there are numerous works on the phenomenon of DDT, the mechanism of this process is yet to be fully understood with broad consensus. Therefore, it is deemed that further study is required to clarify the mechanism in the DDT.

It may be reasonable to consider that the main controlling factors in sustained detonation downstream and those in DDT generation are essentially the same. Self-sustained detonation may decay to the deflagration state if the conditions for detonation are not satisfied. On the DDT occurrence, there have been several theoretical, experimental and numerical studies published in the literature $[4,5]$.

On the theoretical work, Zeldovich et al. [6] proposed the gradient mechanism of reactions for DDT occurrence; they found that there is an appropriate magnitude of temperature gradient for DDT to happen. Lee et al. [7] described the SWACER (Shock wave acceleration coherent energy release) mechanism, in which the shock-flame interaction allows for a large energy release and hence form a concentration gradient in the induction zone; this gradient promotes the occurrence of detonation. These (so called gradient) mechanisms have been used to explain the DDT phenomena for several cases, but it is still not satisfactory for general cases [8]. Next, Brailovsky and Sivashinsky [9] proposed that the wall friction may be a means to enhance DDT. It is shown that the friction resistance causes a gradual precompression and preheating of the unburned gas adjacent to the advancing deflagration. This leads to a localized thermal explosion which may trigger the occurrence of DDT. However, it is also found that detonation can also be formed in unconfined regions in the absence of wall [10]. Jiang et al.'s [11] study showed that detonation can be sustained in a diverging cylindrical detonation via the generation of new transverse waves. In these unconfined detonations, wall friction is not considered. As such, heating associated with wall friction is not the main reason for DDT to occur.

Still, Vasil'ev [12] proposed a criterion for DDT and obtained the critical Mach number. This criterion is independent of the activation energy. Silvestrini et al. [13] suggested some simplified formulas for evaluating the flame speed and DDT run-up distance of flammable mixtures, based on a large quantity of experimental data. Oran and Gamezo [5] summarized the numerical simulations and experimental data and suggested that the interaction of flame and shock wave is the key to the DDT process. The role of turbulence in DDT is to create conditions in nearby unreacted fuel mixtures that lead to 
ignition centers or "hot spots". It is possible that there may be several different ways of achieving DDT, depending on the parameters of the mixture and the flow condition$s[4,5]$. However, the main or primary parameters to influence the DDT generation are not shown conclusively, and the formation mechanism of the DDT is still not clear.

There are several experimental studies in the literature. Smirnov and Tyurnikov [14] found the following mechanisms of detonation origin based on numerous experiments carried out for hydro-carbon-air mixtures under different conditions. These are:

(1) The detonation wave originates on the flame front immediately.

(2) The detonation wave originates between the shock wave and the flame on the contact surface (which lies between the leading shock wave and the flame front), and is formed as a result of the interaction of the shock waves.

(3) The detonation wave originates between the primary wave and the flame zone in the mixture combustion zone, arising from the contact surface, and formed as a result of the interaction of the primary waves.

(4) The denotation wave originates as a result of the volume spontaneous ignition of compressed gas between the primary wave and the flame front.

Thus, DDT can be generated or enacted with different origins and ways depending on the fuel parameters and the flow conditions.

On other further experiments, Dorofeev et al. [15] proposed a correlation on the characteristic length of blockage for successful DDT to the detonation cell size for a variety of mixtures. It is shown that the distance between orifices of obstacles required for DTT will increase with the cell size. Meyer et al. [16] carried out experiments on a number of deflagration-to-detonation (DDT) enhancement techniques for use in a $\mathrm{H}_{2}$ / Air pulseddetonation engine (PDE). The geometrical configurations include Shchelkin spiral, an extended cavity/spiral, and a coannulus. It is found that DDT is initiated primarily through local explosions that are highly dependent on the particular geometry. In addition to various geometries, the effect of equivalence ratio and spark timing are also investigated. $\mathrm{Li}$ et al. [17] carried out experimental study on the influence of the tube diameter on DDT run-up distance. It is found that the tube diameter effect is associated with the amplification, flame acceleration and heat loss. It is also related to the properties of the mixtures. Zhu et al. [18] found from the experiments that there is a critical high speed deflagration that propagates at about $1 / 2 U_{c j}$ before the onset of detonation. This critical $1 / 2 U_{c j}$ velocity is independent of the turbulence parameters and depends only on the energetics of the mixture like a CJ detonation. Sorin et al. [19] showed that the run-up distance of DDT decreases with the reduced activation energy and on the heat release. Aizawa et al. [20] found that turbulence may have some influence on the DDT run-up distance when $R e$ is larger than a certain range of values for the hydrogen/air mixture flow. Lee et al. [21] found that obstacles in the channel play a major role in generating small/large scale turbulence that enhances flame acceleration. Teodorczyk et al.'s [22] experiments showed that obstacles giving rise to high channel blockage ratio are destructive for the 
flame propagation and regardless of the beneficial effect arising from turbulence generation on the DDT. New et al. [23] carried out a series of experiments for various geometric DDT devices and showed that Shchelkin spiral is observed to be the best performer among the DDT devices. They also found that convergent-divergent throat configurations have a tendency to destabilize the coupling between the flame and shock fronts.

For numerical study, Tegnér and Sjögreen [24] did simulations for the DDT process by solving the 2D Navier-Stokes equations with a one-step reaction model. It was found that for stiff and non-stiff problems, the DDT process is different. There are three stages of the DDT. These are:

(1) A shock wave firstly develops ahead of the reaction zone. Both the strength of the shock wave and the rate of reaction in the flame increase gradually.

(2) When the shock wave reaches a sufficiently high level, the peak pressure is larger than the ZND pressure, and this pressure leads to the reaction zone connecting to the shock wave.

(3) This event results in an overdriven detonation; then, this overdriven detonation relaxes to the $\mathrm{CJ}$ detonation.

Parra-Santos et al. [25] did numerical simulation of DDT using the 1D Euler equation. The run-up distance of DDT is calculated and qualitative agreement with experimental data is obtained. They concluded that the interaction of the flame front and the pressure wave is primarily responsible for the DDT. The result from this simulation using Euler equation is essentially the same as that of Tegnér and Sjögreen [24] using the 2D NavierStokes equations.

Vaagsaether et al. [26] simulated the DDT in a circular tube using a turbulence model and obtained interesting result. The calculation was performed in an axisymmetrical cylindrical coordinates. They found that the obstruction in the channel helps the DDT since it creates high pressures in the ignition end of the tube and very high gas velocities at and behind the obstruction opening. Lu et al. [27] found through their 2D simulation that detonation could be self ignited in a wedged channel under certain range of incoming Mach number and/or wedge angle. This result may be related to the mechanism of DDT devices using obstacles since obstacles create shock waves in a similar ways. Gamezo et al.'s [28] simulation results for obstructed channel showed that various instabilities can occur in that channel which enhance the shock strength. The collision of the shock waves with the obstacles can promote the DDT.

In this study, numerical simulation is carried out to investigate the process of DDT occurring in a straight duct. In particular, effects of energy release of the mixture on the DDT are studied. The governing equations are the Euler equations and the one-step Arrhenius chemical reaction model for premixed fuel. The parameters used in this study are as follows: the specific ratio 1.20, and the activation temperature of $T i=15$, while the value of the energy release is changed. 


\section{Governing equations}

The program used for present study is a 3D Euler code developed previously. We take the grid number in the $z$ direction as unity to simulate the 2D problems. The governing equations describing the fluid flow and the detonation propagation are the three-dimensional Euler equations with a source term which represents the chemical reaction process. In conservative form, these are written as [29-31]

$$
\frac{\partial U}{\partial t}+\frac{\partial F}{\partial x}+\frac{\partial G}{\partial y}+\frac{\partial H}{\partial z}=S,
$$

where the conserved variable vector $U$, the flux vectors $F, G$, and $H$ as well as the source vector $S$ are given, respectively, as

$$
U=\left[\begin{array}{c}
\rho \\
\rho u \\
\rho v \\
\rho w \\
E \\
\rho Y
\end{array}\right], \quad F=\left[\begin{array}{c}
\rho u \\
\rho u^{2}+p \\
\rho u v \\
\rho u w \\
(E+p) u \\
\rho u Y
\end{array}\right], G=\left[\begin{array}{c}
\rho v \\
\rho u v \\
\rho v^{2}+p \\
\rho v w \\
(E+p) v \\
\rho v Y
\end{array}\right], \quad H=\left[\begin{array}{c}
\rho w \\
\rho u w \\
\rho v w \\
\rho w^{2}+p \\
(E+p) w \\
\rho w Y
\end{array}\right], \quad S=\left[\begin{array}{c}
0 \\
0 \\
0 \\
0 \\
0 \\
\omega
\end{array}\right] .
$$

Here $u, v$, and $w$ are the components of the fluid velocity in the $x, y$, and $z$ directions, respectively, in the Cartesian coordinates system, $\rho$ is the density, $p$ is the pressure, $E$ is the total energy per unit volume, and $Y$ is the mass fraction of the reactant. The total energy $E$ is defined as

$$
E=\frac{p}{\gamma-1}+\frac{1}{2} \rho\left(u^{2}+v^{2}+w^{2}\right)+\rho q Y,
$$

where $q$ is the heat production of reaction, and $\gamma$ is the ratio of specific heats. The source term $\omega$ is assumed to be in an Arrhenius form

$$
\omega=-K \rho Y e^{-\left(T_{i} / T\right)},
$$

where $T$ is the temperature, $T_{i}$ is the activation temperature, and $K$ is a constant rate coefficient. For a perfect gas, the state equation is

$$
p=\rho R T \text {. }
$$

As such, Eqs. (2.1) to (2.5) constitute a closed system of equations. This system of equations can be solved using a proper numerical technique.

The above mentioned equations are made dimensionless based on the state of the unburned gas,

$$
\begin{array}{llllll}
\bar{\rho}=\frac{\rho}{\rho_{0}}, & \bar{p}=\frac{p}{p_{0}}, & \bar{T}=\frac{T}{T_{0}}, & \bar{u}=\frac{u}{u_{0}}, & \bar{v}=\frac{v}{u_{0}}, & \bar{w}=\frac{w}{u_{0}}, \\
\bar{x}=\frac{x}{x_{0}}, & \bar{t}=\frac{t}{t_{0}}, & \bar{E}=\frac{E}{p_{0}}, & \bar{K}=\frac{K x_{0}}{u_{0}}, & \bar{q}=\frac{q}{u_{0}{ }^{2}}, & \bar{T}_{i}=\frac{T_{i}}{T_{0}},
\end{array}
$$


where $u_{0}=\sqrt{R T_{0}}$ and $t_{0}=x_{0} / u_{0}$. The reference length $x_{0}$ is chosen as the half-reaction length $(L)$, which is defined as the distance between the detonation front and the point where half of the reactant is consumed by chemical reaction in ZND detonation. This reference length is taken as the unit length to normalize the computational domain. Because of the self-similarity of the Euler equations, its dimensionless form and its original form is identical. For convenience, the overbar on each variable is dropped in the following sections. In this study, the one-step model of reaction is selected to reveal essential detonation physics not encumbered by complex chemical kinetics. In addition, this model permits the variation of the activation energy, which is able to model the rate of reaction of different fuel mixtures in the study of DDT.

\section{Numerical implementation}

The system of conservation laws of inviscid fluid combined with the one-step chemical reaction model are discretized spatially in the eigenvector space in Cartesian coordinates using the fifth-order WENO (Weighted Essentially NonOscillatory) scheme, and the final discretized variables are solved with a 3rd order TVD (Total Variation Diminishing) Runge-Kutta method in time [29,30]. The computer code for the pure Euler flow (no chemical reaction) has been validated for the steady flow of oblique shock wave past a wedge, and the unsteady flow in one-dimensional shock tubes for both the so called Lax and Sod problems. For all the three examples above, the accuracy of the simulated results is deemed good as these results are in agreement with analysis and other works. Interested readers may refer to $[29,30]$ for details. Other comparisons with analysis are made for the 1D simulation of detonation again with good concurrence. The 3D version of this program has been also used to simulate 3D detonations [29-31], and grid convergence has been confirmed.

For the combustion model, the controlling parameters are the ratio of specific heats $\gamma$, the heat release per unit mass of fuel $q$, and the activation temperature $T_{i}$. One additional free parameter, the reaction-rate pre-exponential factor $K$, sets the spatial and temporal scales.

The computational domain is a plane duct. The width of the duct is selected to be the order of the cell width (as is well known in the literature, the value of cell width varies with the activation temperature and other parameters). The left end of the duct is closed while the right end is open. A spark with high energy is placed at the location near the left end. Initially, the pressure and temperature within the spark are given as constants. In this study, the values of spark pressure $p_{s}$ and the spark temperature are selected according to a ratio of them to the CJ state. At $t=0$, this spark expands radially outwards. At the downstream of the duct, shock wave and flame front may be formed. As observed, the generation of DDT at downstream will depend on the evolution of the two fronts.

The size of the computational duct is selected as follows. The dimensionless size of 
the duct is $30 \times 320$, and the grid points are 150 and 3200 in $x$ and $y$ directions, respectively. The following boundary conditions are employed. The upper and bottom walls and the left boundary are set with reflective boundary conditions. The right boundary is given by the un-burnt quiescent mixture parameter at atmospheric condition.

\section{Results and discussions}

Fig. 1 shows the simulation results with various values of energy release for $T i=15$ for various values of energy release. It can be seen that the run-up distance of DDT is increased with energy release for $q=50 \sim 20$, and is reduced for $q=20 \sim 5$. This complex phenomenon is difficult to be explained. Sorin et al. [19] found through experiments of energy release $q=50 \sim 20$ that the DDT distance decreased with the increase of energy release, which is in agreement with the present simulation of the energy release range. However, for energy release $q=20 \sim 5$, there is no experimental data to be compared. It is reckoned that the physics behind this phenomenon may be related to the mechanism of detonation stability. In [33], the linear stability analysis for one-dimensional (1D) detonation found that the detonation is unstable at the middle range of energy release at a given $T i$ (if $T i>14$ ), see Fig. 2. For larger and smaller energy release, the detonation is
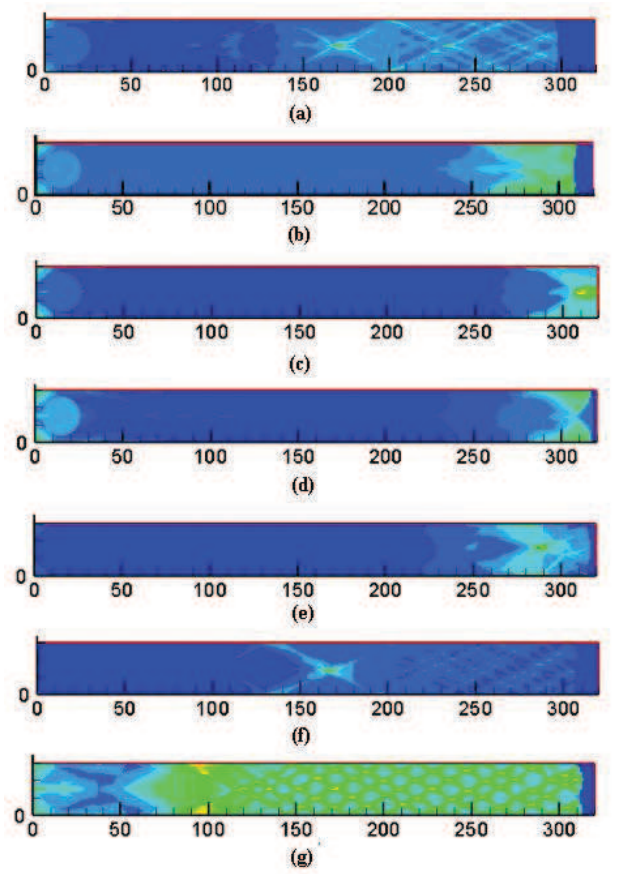

Figure 1: Flow pattern with record of maximum pressure for activation temperature $T i=15$ and specific heat ratio $\gamma=1$.2. The heat release are (a) $q=50, t=58$; (b) $q=30, t=109$; (c) $q=25, t=136$; (d) $q=20, t=154$; (e) $q=15, t=143$; (f) $q=10, t=119$; (g) $q=5, t=127$. 


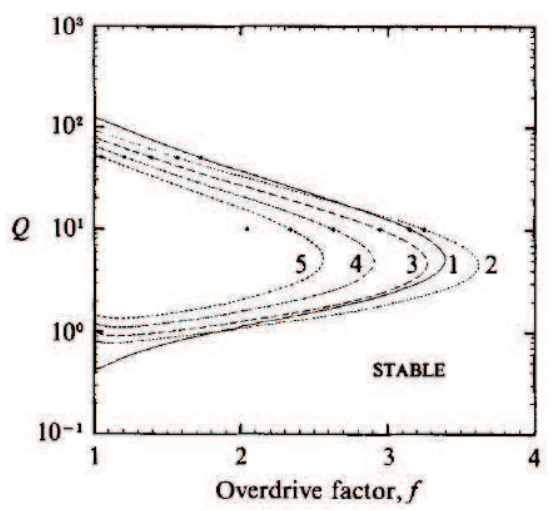

Figure 2: The neutral stability curves in an $(f, Q)$-plane for specific heat ratio $\gamma=1.2$ and activation temperature $E=50$. The $f$ stands for the overdriven factor. The curves are numbered according to increasing frequency of perturbation. This picture is adapted from Lee and Stewart (JFM, 1990).
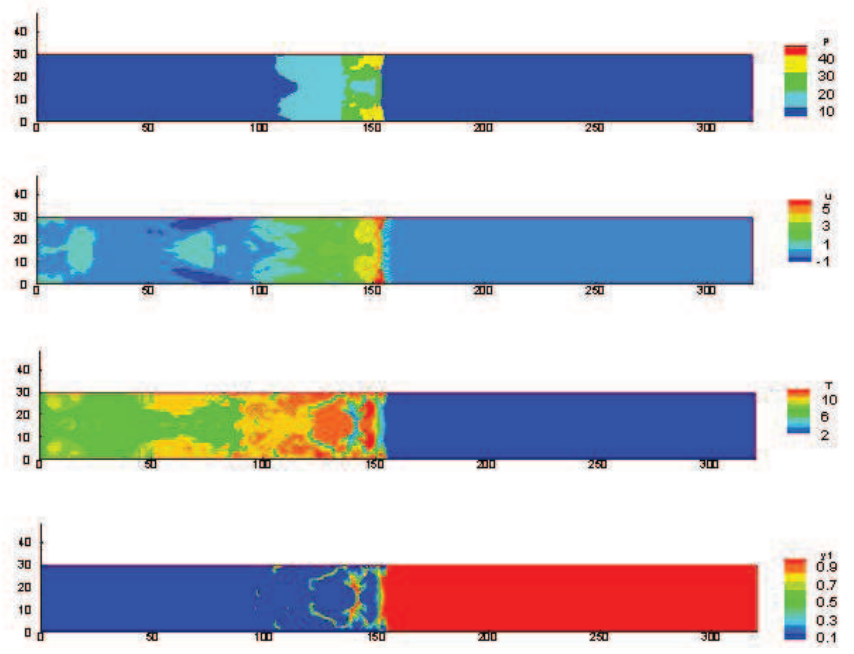

Figure 3: Flow contours for activation temperature $T i=15$, heat release $q=50$ and specific heat ratio $\gamma=1.2$. The variables are pressure $(p)$, velocity $(u)$, temperature $(T)$ and mass fraction of fuel $\left(Y_{1}\right)$. The dimensionless time from the start is $t=38.4$.

more stable. It is seen that this tendency of stability with the energy release is similar to our simulation results.

The physical phenomena in nature may have their similarity in common. In turbulent researches, it has been found that the factors affecting the laminar to turbulent flow transition also influence the sustenance of turbulence. In turbulent transition and full developed turbulence, the factors result in turbulent transition also can maintain the sustenance of turbulence, and vice versa. In DDT occurrence, it is presumed that the factors strengthening the DDT should also help the sustenance and stability of detonation. The above analysis shows that the simulation results of DDT for varying energy release in present study are in agreement with the stability characteristic from mathematical stabil- 

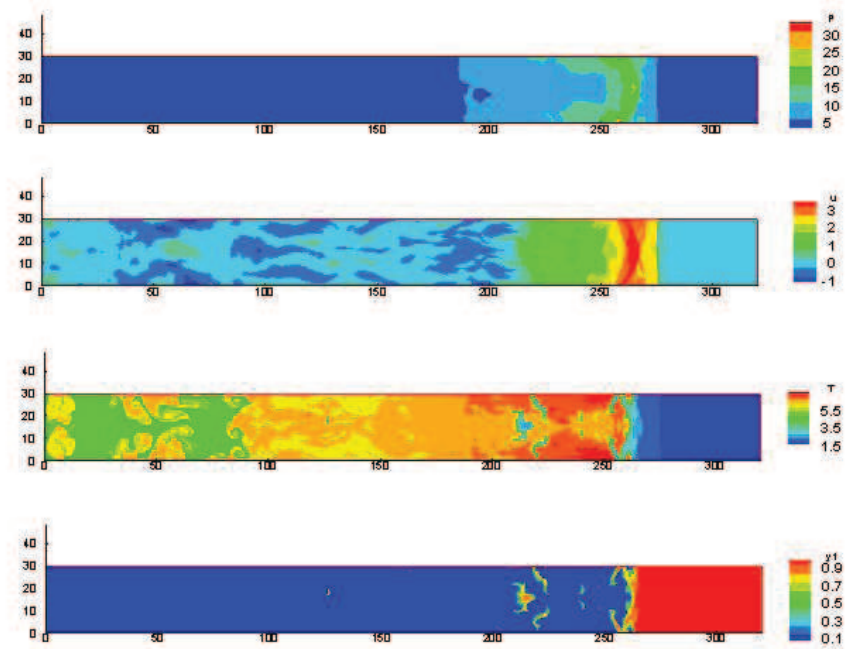

Figure 4: Flow contours for activation temperature $T i=15$, heat release $q=30$ and specific heat ratio $\gamma=1.2$. The variables are pressure $(p)$, velocity $(u)$, temperature $(T)$ and mass fraction of fuel $\left(Y_{1}\right)$. The dimensionless time from the start is $t=100.4$.

ity theory in [33].

Fig. 3 shows the contour plots for $q=50$ and $T i=15$. In this case, the shock wave and the flame front are decoupled in the early stage. However, owing to the high pressure of the shock wave, the fuel between the two fronts is heated and compressed, and then selfignited at further downstream behind the shock wave. It can be seen from the contours of the temperature $(T)$ and mass fraction of fuel $(Y)$ in Fig. 3 that several self-ignited areas are observed. These self-ignited flames connect the shock wave and the flame front, and thus the shock wave and flame front are merged to form one front. Finally DDT is generated due to the high pressure and temperature behind the shock wave. This phenomenon discussed is in agreement with the experimental finding as described by Smirnov and Tyurnikov [14].

Figs. 4 to 7 show the contour plots for $q=30,20,10$, and 5 at $T i=15$, respectively. For these cases, the DDT is not generated immediately but at a short distance after the ignition starting. The shock wave and the flame front are decoupled and this distance between them becomes larger (monotonically) on moving further downstream. At further downstream, however, owing to the rise of the temperature at the flame front, which is swept by the leading shock wave, the reaction at the flame front becomes more intense at the flame front vicinity. This will in turn cause the pressure to increase around the flame front. This pressure wave interacts with the flame, and finally it leads to the flame front accelerating. In these pictures, it can be seen that the second pressure wave is formed due to intensive reaction ( $p$ contour), whereas the flame is not accelerated yet. Finally, the flame front eventually catches up with the leading shock wave and the DDT is thus formed. This phenomenon is similar to the mechanism (3) as described by Smirnov 

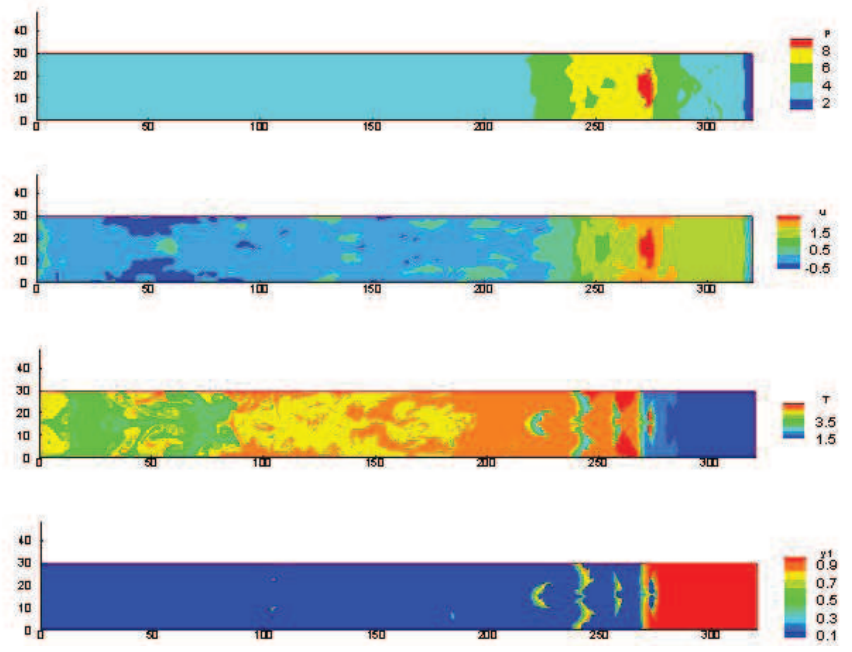

Figure 5: Flow contours for activation temperature $T i=15$, heat release $q=20$ and specific heat ratio $\gamma=1.2$. The variables are pressure $(p)$, velocity $(u)$, temperature $(T)$ and mass fraction of fuel $\left(Y_{1}\right)$. The dimensionless time from the start is $t=141.6$.
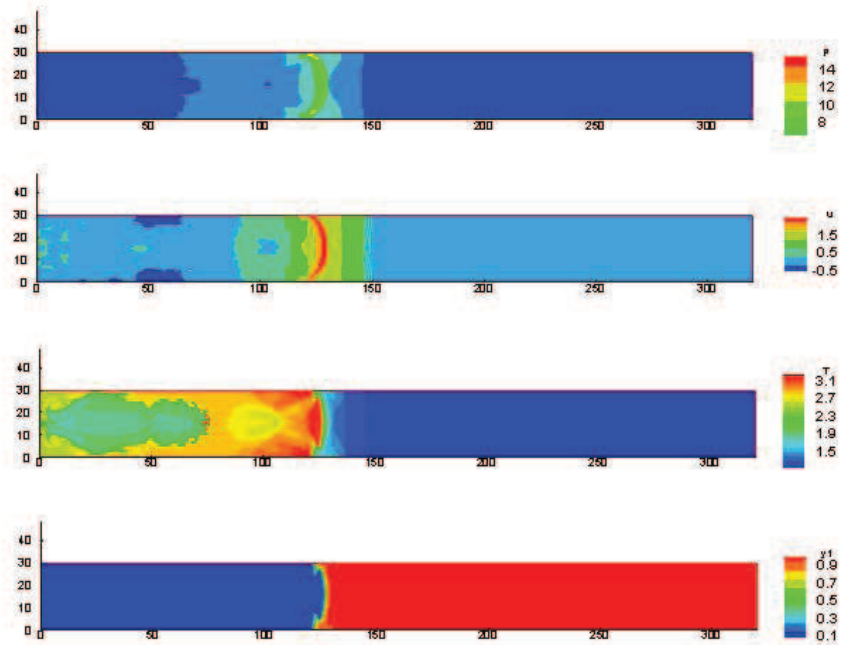

Figure 6: Flow contours for activation temperature $T i=15$, heat release $q=10$ and specific heat ratio $\gamma=1.2$. The variables are pressure $(p)$, velocity $(u)$, temperature $(T)$ and mass fraction of fuel $\left(Y_{1}\right)$. The dimensionless time from the start is $t=70.9$.

and Tyurnikov [14]. Liberman et al.'s study showed that the formation of a preheated zone ahead of propagating flame controls the DDT process [34]. This fact reflects that the interaction of the shock wave and the flame front plays an important role in the DDT occurrence.

Fig. 8 shows the distributions of the flow and reaction parameters along the centerline of the channel, which corresponds to the Fig. 3. It can be seen that the flame surface (peak 


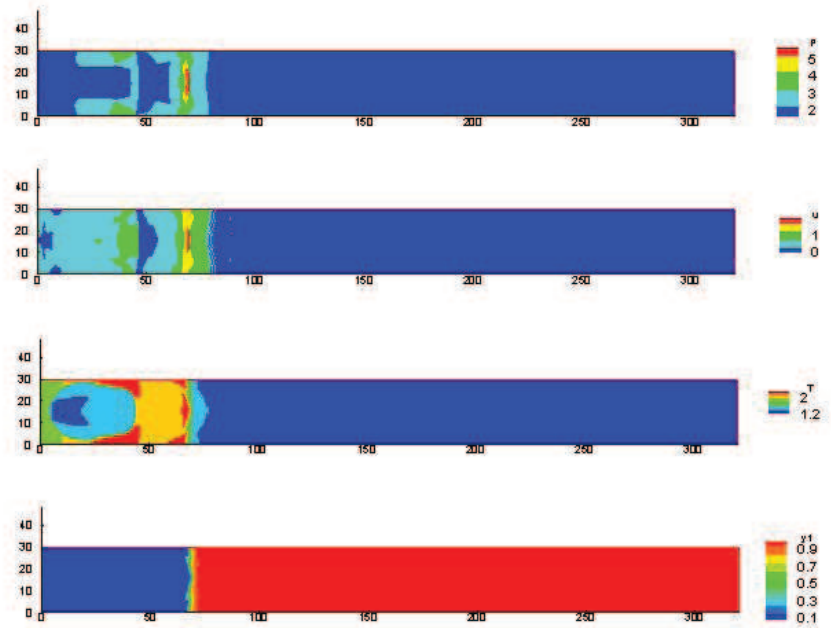

Figure 7: Flow contours for activation temperature $T i=15$, heat release $q=5$ and specific heat ratio $\gamma=1.2$. The variables are pressure $(p)$, velocity $(u)$, temperature $(T)$ and mass fraction of fuel $\left(Y_{1}\right)$. The dimensionless time from the start is $t=35.6$.

of $T$ ) is apart from the leading shock wave (peaks of $p$ and $u$ ) at the earlier time (Figs. 8(a) and (b)), and the distance between them becomes short with the time evolution (Figs. 8(c) and (d)). Simultaneously, the pressure and the velocity at the flame front increase with the moving the flame front. Finally, at a sufficient long distance, the two fronts meet together and the DDT occurs. In this process, it is clearly seen that the interaction of the two fronts plays important role for the DDT to happen.

As is generally found, the whole process of DDT generally includes two stages: (1) the flame acceleration and (2) deflagration and deflagration-to-detonation transition. In these two stages, the heat release from the reaction has different effects on propagation mechanisms of combustion wave and DDT occurrence. In the first stage (flame acceleration), the reaction heat makes the fluid expansion and thus leads to combustion wave accelerating (Figs. 8(a) and (b)). In the second stage (DDT), the reaction heat makes the pressure at the flame front fluid become high and the velocity become large. Thus, this results in the flame front to catch up the leading shock wave and finally the DDT is formed (Figs. 8(c) and (d)).

\section{Conclusions}

Numerical study for the DDT process is carried out for different values of the energy release. In particular, effect of energy release on the DDT process is studied. The model parameters used are the heat release at $q=50,30,25,20,15,10$ and 5, respectively, the specific heat ratio is at 1.2 , and the activation temperature at $T i=15$. For all the cases, the initial energy in the spark is about the same compared to the detonation energy at the Chapman-Jouguet (CJ) state. 


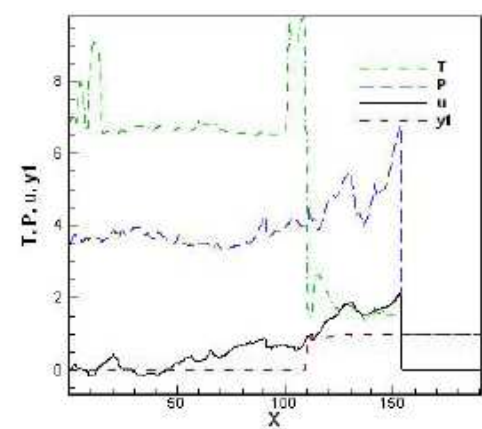

(a) $t=50$

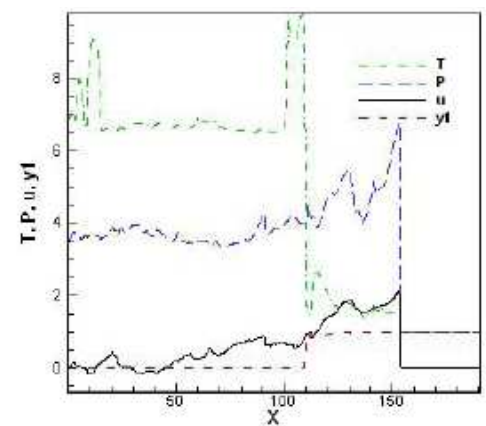

(c) $t=101$

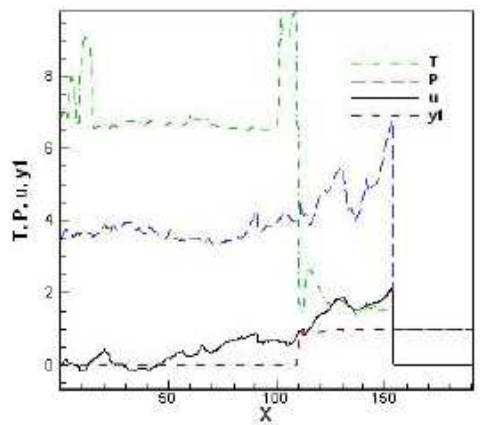

(b) $t=76$

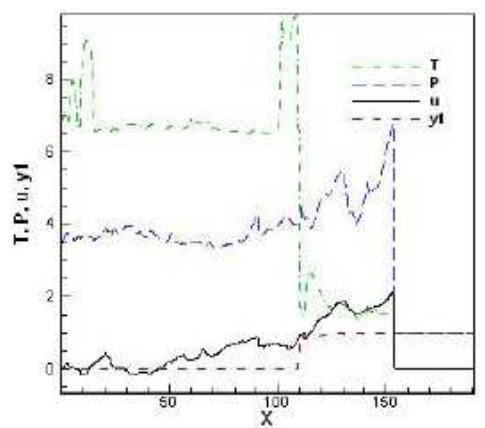

(d) $t=116$

Figure 8: Distributions of the flow and combustion parameters at the centerline of the channel, for activation temperature $T i=15$, spark pressure $p_{s}=15$, and $Q=50$. The variables are pressure $(p)$, velocity $(u)$, temperature $(T)$ and mass fraction of fuel $\left(Y_{1}\right)$.

It is found from the simulation that the DDT occurrence strongly depends on the magnitude of the energy release. The run-up distance of DDT decreases with the increase of the energy release for $q=50 \sim 20$, and increases with the increase of the energy release for $q=20 \sim 5$ at the condition of constant activation temperature. The present study shows that the simulation results of DDT for varying energy release are in agreement with the stability characteristic from mathematical stability theory.

In all the cases, the interaction of the shock wave with the flame front plays an important role. This kind of interaction results in the formation of hot spots as well as the flame acceleration, and finally leads to DDT.

Finally, similar to turbulence physics, the factors to strengthen the DDT would make the detonation more stable, and vice versa.

\section{Acknowledgements}

This work is supported by Science Foundation of Zhejiang Sci-Tech University (ZSTU) under Grant No. 11130032241201 and the opening research foundation of State Key Labo- 
ratory of High Temperature Gas Dynamics in Institute of Mechanics of Chinese Academy of Sciences.

\section{References}

[1] K. KaIlASANATH, Review of propulsion applications of detonation waves, AIAA J., 38 (2000), pp. 1698-1708.

[2] F. LU AND R. Bellini, Progress in modeling pulse detonations, Lecture Notes in Workshop on Moving Interface Problems and Applications in Fluid Dynamics, 8 January-31 March 2007, IMS, NUS.

[3] L. Bédard-Tremblaya, L. Fanga, L. Bauwens, Z. Chengb and A. V. Tchouvelev, Numerical simulation of hydrogenCair detonation for damage assessment in realistic accident scenarios, J. Loss Prevention in the Process Industries, 21 (2008), pp. 154-161.

[4] G. Ciccarelli AND S. Dorofeev, Flame acceleration and transition to detonation in ducts, Progress in Energy and Combustion Science, 34 (2008), pp. 499-550.

[5] E. S. ORAN AND V. N. GAMEZO, Origins of the deflagration-to-detonation transition in gas-phase combustion, Combust. Flame, 148 (2007), pp. 4-47.

[6] Ya. B. Zeldovich, V. B. Librovich, G. M. MaKhViladZe and G. I. Sivashinsky, On the development of detonation in a non-uniformly preheated gas, Astron. Acta, 15(5-6) (1970), pp. 313-321.

[7] J. H. Lee, R. KnYstautas And N. YoshiKawa, Photochemical initiation of gaseous detonations, Acta Astronautica, 5 (1978), pp. 971-982.

[8] A. L. Kapila, D. W. Schwendman, J. J. QUiRK AND Y. HaWA, Mechanisms of detonation formation due to a temperature gradient, Combust. Theory Model., 6 (2002), pp. 553-594.

[9] I. BRAILOVSKYA AND G. I. SIVASHINSKY, Hydraulic resistance as a mechanism for deflagrationto-detonation transition, Combust. Flame, 122 (2000), pp. 492-499.

[10] A. M. KHOKhlov, E. S. ORAn AND J. C. WheELER, A theory of deflagration-to-detonation transition in unconfined flames, Combust. Flame, 108(4) (1997), pp. 503-517.

[11] Z. L. JIANG, G. L. HAN, C. WANG AND F. ZHANG, Self-organized generation of transverse waves in diverging cylindrical detonations, Combust. Flame, 156(8) (2009), pp. 1653-1661.

[12] A. A. VASIL'EV, Estimation of critical conditions for the detonation-to-deflagration transition, Combustion Explosion and Shock Waves, 42 (2006), pp. 205-209.

[13] M. Silvestrini, B. GeNOVA AND G. PARISI, Flame acceleration and DDT run-up distance for smooth and obstacles filled tubes, J. Loss Prevention in the Process Industries, 21 (2008), pp. $555-562$.

[14] N. N. SMIRNOV AND M. V. TYURNIKOV, Experimental investigation of deflagration to detonation transition in hydrocarbon-air gaseous mixtures, Combust. Flame, 100 (1995), pp. 661-668.

[15] S. B. Dorofeev, V. P. Sidorov, M. S. KuZnetsov, I. D. Matsukov, And V. I. AleKseev, Effect of scale on the onset of detonations, Shock Waves, 10 (2000), pp. 137-149

[16] T. R. Meyer, J. L. HOKE, M. S. BROWN, J. R. GORD, AND F. R. SCHAuer, Experimental study of deflagration-to-detonation enhancement techniques in a $h_{2}$ /air pulsed-detonation engine, AIAA-2002-3720, 2002.

[17] J. LI, W. H. LAI AND K. CHUNG, Tube diameter effect on deflagration-to-detonation transition of propane-oxygen mixtures, Shock Waves, 16 (2006), pp. 109-117.

[18] Y. J. ZHU, J. CHAO AND J. H. S. LEE, An experimental investigation of the propagation mechanism of critical deflagration waves that lead to the onset of detonation, Proc. Combust. Inst., 31 
(2007), pp. 2455-2462.

[19] R. SORIN, R. Zitoun AND D. Desbordes, Optimization of the deflagration to detonation transition: reduction of length and time of transition, Shock Waves, 15 (2006), pp. 137-145.

[20] K. Aizawa, S. Yoshino, T. Mogi, H. Shina, Y. Ogata, Y. WAdA And A. K. Hayashi, Study of detonation initiation in hydrogen/air flow, Shock Waves, 18 (2008), pp. 299-305.

[21] S. Y. Lee, J. Watts, S. SARetTo, S. Pal, C. Conrad, R. WoodWARD, AND R. SAntoro, Deflagration to detonation transition processes by turbulence-generating obstacles in pulse detonation engines, J. Propulsion Power, 20 (2004), pp. 1026-1036.

[22] A. TEODORCZYK, P. DROBNIAK AND A. DABKOWSKI, Fast turbulent deflagration and DDT of hydrogen-air mixtures in small obstructed channel, Inter. J. Hydrogen Energy, 34 (2009), pp. 5887-5893.

[23] T. NeW, P. K. PANiCKer, K. ChUI, H. TSAI, AND F. K. LU, Experimental study on deflagrationto-detonation transition enhancement methods in a PDE, AIAA-2006-7958, 14th AIAA/AHI Space Planes and Hypersonic Systems and Technologies Conference, November 6-9, 2006, Canberra, Australia.

[24] J. TEGNÉR, AND B. SJÖGREEN, Numerical investigation of deflagration to detonation transitions, Combust. Sci. Tech., 174 (2002), pp. 153-186.

[25] M. T. PARRA-SANTOS AND F. CASTRO-RUIZ, Numerical simulation of the deflgration to detonation transition, Combustion, Explosion, and Shock Waves, 41 (2005), pp. 215-222.

[26] K. VAAGSAETHer, V. KNUdSEN AND D. BJERKETVEDT, Simulation of flame acceleration and DDT in $\mathrm{H}_{2}$-air mixture with a flux limiter centered method, Int. J. Hydrogen Energy, 32 (2007), pp. 2186-2191.

[27] F. K. LU, H. Y. FAN AND D. R. WILSON, Detonation waves induced by a confined wedge, Aero. Sci. Tech., 10 (2006), pp. 679-685.

[28] V. N. GAMEZO, T. OGAWA AND E. S. ORAN, Numerical simulations of flame propagation and DDT in obstructed channels filled with hydrogen-air mixture, Proc. Combust. Inst., 31 (2007), pp. 2463-2471.

[29] H.-S. DOU, H. M. TSAI, B. C. KHOO AND J. QIU, Simulations of detonation wave propagation in rectangular ducts using a three-dimensional WENO scheme, Combust. Flame, 154 (2008), pp. 644-659.

[30] H.-S. DOU AND B. C. KHOO, Effect of initial disturbance on the detonation front structure of a narrow duct, Shock Waves, 20(2) (2010), pp. 163-173.

[31] H.-S. DOU, H. M. TSAI, B. C. KHOO AND J. QIU, Three-dimensional simulation of detonation waves using WENO schemes, 45th AIAA Aerospace Sciences Meeting and Exhibit, Reno, Nevada, 8-11 January 2007. (AIAA Paper-2007-1177).

[32] H.-S. DOU, B. C. KHOO AND H. M. TSAI, Physics of detonation captured by three-dimensional simulations, Proceedings of the 26th International Symposium on Shock Waves, July 15-20, 2007, Goettingen, Germany, Springer-Verlag.

[33] H. I. LEE AND D. S. STEWART, Calculation of linear detonation instability: one-dimensional instability of plane detonation, J. Fluid Mech., 216 (1990), pp. 103-132.

[34] M. A. LibERMAN, M. KuZNeTSOV, A. IVANOV AND I. MATSUKOV, Formation of the preheated zone ahead of a propagating flame and the mechanism underlying the deflagration-to-detonation transition, Phys. Lett. A, 373(5) (2009), pp. 501-510. 